\title{
A novel drug-phospholipid complex enriched with micelles: preparation and evaluation in vitro and in vivo
}

This article was published in the following Dove Press journal:

International Journal of Nanomedicine

I February 2013

Number of times this article has been viewed

\author{
Hai-jian Xia ${ }^{1,2}$ \\ Zhen-hai Zhang' \\ Xin Jin' \\ Qin $\mathrm{Hu}^{\prime}$ \\ Xiao-yun Chen' \\ Xiao-bin Jia'
}

'Key Laboratory of New Drug Delivery System of Chinese Materia Medica, Jiangsu Provincial Academy of Chinese Medicine, Nanjing, China; ${ }^{2}$ College of Pharmacy, Nanjing University of Chinese Medicine, Nanjing, China
Correspondence: Xiao-bin Jia Key Laboratory of New Drug Delivery System of Chinese Materia Medica, Jiangsu Provincial Academy of Chinese Medicine, 100 Shizi Road, Nanjing, Jiangsu 210028, People's Republic of China

Tel +862585608672

$\mathrm{Fax}+862585637809$

Email xiaobinjia_nj@I26.com
Abstract: Mixed micelles are widely used to increase solubility and bioavailability of poorly soluble drugs. One promising antitumor drug candidate is 20 (S)-protopanaxadiol (PPD), although its clinical application is limited by low water solubility and poor bioavailability after oral administration. In this study, we developed mixed micelles consisting of PPD-phospholipid complexes and Labrasol ${ }^{\circledR}$ and evaluated their potential for oral PPD absorption. Micelles were prepared using a solvent-evaporation method, and their physicochemical properties, including particle size, zeta potential, morphology, crystal type, drug loading, drug entrapment efficiency, and solubility, were characterized. Furthermore, in vitro release was investigated using the dialysis method, and transport and bioavailability of the mixed micelles were investigated through a Caco-2 cell monolayer and in vivo absorption studies performed in rats. Compared with the solubility of free PPD $(3 \mu \mathrm{g} / \mathrm{mL})$, the solubility of PPD in the prepared mixed micelles was $192.41 \pm 1.13 \mu \mathrm{g} / \mathrm{mL}$ in water at room temperature. The in vitro release profiles showed a significant difference between the more rapid release of free PPD and the slower and more sustained release of the mixed micelles. At the end of a 4-hour transport study using Caco-2 cells, the apical-to-basolateral apparent permeability coefficients $\left(\mathrm{P}_{\text {app }}\right)$ increased from $(1.12 \pm 0.21) \times 10^{6} \mathrm{~cm} / \mathrm{s}$ to $(1.78 \pm 0.16) \times 10^{6} \mathrm{~cm} / \mathrm{s}$, while the basolateral-to-apical $\mathrm{P}_{\text {app }}$ decreased from $(2.42 \pm 0.16) \times 10^{6} \mathrm{~cm} / \mathrm{s}$ to $(2.12 \pm 0.32) \times 10^{6}$. In this pharmacokinetic study, compared with the bioavailability of free PPD (area under the curve $[\mathrm{AUC}]_{0-\infty}$ ), the bioavailability of PPD from the micelles ( $\mathrm{AUC}_{0-\infty}$ ) increased by approximately $216.36 \%$. These results suggest that novel mixed micelles can significantly increase solubility, enhance absorption, and improve bioavailability. Thus, these prepared micelles might be potential carriers for oral PPD delivery in antitumor therapies.

Keywords: 20(S)-protopanaxadiol, phospholipid complex, Labrasol, mixed micelles, Caco-2 cell monolayer, bioavailability

\section{Introduction}

The substance 20(S)-protopanaxadiol (PPD) is mainly derived from ginseng and is an aglycon of the PPD-containing ginsenosides, such as ginsenoside $\mathrm{Rb} 1, \mathrm{Rb} 2, \mathrm{Rb} 3$, Rh2, and Rg3 (Figure 1). Moreover, it is the main metabolite of the PPD-type ginsenosides as well as the product of their acid hydrolysis. ${ }^{1-5}$ PPD saponins, such as Rg3 and Rh2, have been shown to possess a variety of beneficial effects for antitumor, antifatigue, antiaging, anticancer, and immune regulatory processes. ${ }^{6-8}$ Moreover, the pharmacological effects of PPD are similar to or stronger than those of Rh2 and Rg3. ${ }^{9-11}$ Thus, PPD is a potential therapeutic agent for the prevention and treatment of cancer and tumor. ${ }^{12,13}$ However, its poor solubility and low permeability are 


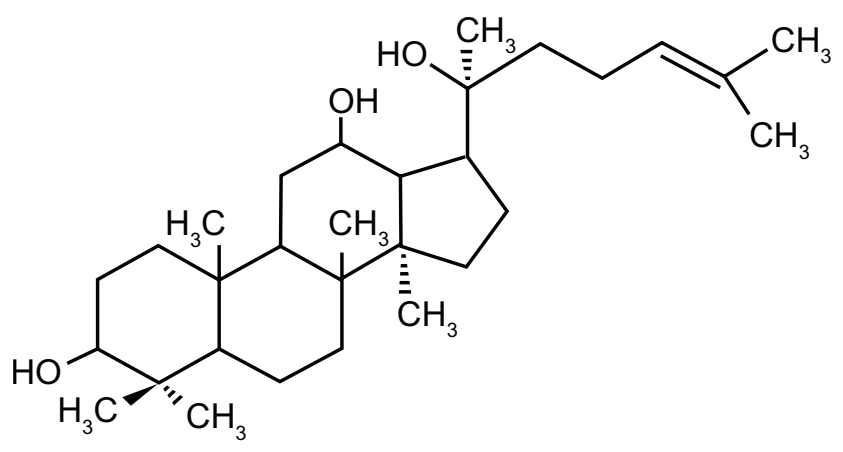

Figure I Chemical structure of 20(S)-protopanaxadiol.

associated with its poor absorption and bioavailability, which hinder its development and therapeutic use. ${ }^{14}$

The poor water solubility of PPD has limited its use, and it is clinically important to resolve this problem. In addition to several traditional approaches, such as the addition of a solubilizer or auxiliary solvent and the use of mixed solvents, methods such as salt formation, pro-drug formation by chemical modification, preparation of solid dispersion, or use of cyclodextrin inclusion compounds, have been employed to improve the solubility of hydrophobic drugs. ${ }^{15-17}$ However, mixed micelles are emerging as novel drug delivery vehicles and have been adopted in recent years to improve the bioavailability and solubility of hydrophobic drugs, thus gaining increasing attention. Moreover, the nanometer size of the micelles contributes to their high stability both in vitro and in vivo, and good biocompatibility. Micelles can solubilize a broad variety of poorly soluble pharmaceuticals, and many of these drug-loaded micelles are currently at different stages of preclinical and clinical trials. ${ }^{18}$

Thus, nanotechnological approaches exhibit considerable potential for increasing solubility and enhancing absorption. It has been reported that the mixed micelles system is one of the most promising strategies to deliver poorly soluble anticancer drugs. ${ }^{19-22}$ To improve the water solubility and therapeutic efficacy of PPD, a novel micelle loaded with PPD-phospholipid complex was prepared using the solvent-evaporation method in this study. Its physical properties were characterized. Transport was examined using the Caco-2 monolayer model, and pharmacokinetic studies in rats were performed, to evaluate the improvement of the solubility, absorption, and bioavailability of the hydrophobic drugs.

\section{Materials and methods Materials}

The materials 20(S)-PPD and ginsenoside-Rh2(S) (purity $>$ 98\%) were purchased from ZeLang Pharmaceuticals
Technology Co, Ltd (Nanjing, China). Soybean phospholipids were purchased from Taiwei Pharmaceutical Co, Ltd (Shanghai China). Labrasol ${ }^{\circledR}$ (Gattefossé, St-Priest, France) was provided by Thanch Pharmaceuticals Technology Co, Ltd (Shanghai, China). Hank's balanced salt solution (HBSS) and purified water from Milli-Q water (EMD Millipore, Billerica, MA, USA) were prepared in the laboratory. Acetonitrile and methanol (Merck and Co, Inc, Whitehouse Station, NJ, USA) were of chromatographic grade. All of the other reagents were analytically pure.

\section{Preparation of the mixed micelles consisting of PPD-phospholipid complexes and Labrasol}

The PPD-phospholipid complexes enriched with micelles were prepared using the solvent-evaporation method. Briefly, PPD and phospholipid were dissolved in $10 \mathrm{~mL}$ of anhydrous ethanol at $50^{\circ} \mathrm{C}$, with gentle agitation $(40 \mathrm{r} / \mathrm{min})$ for about 1 hour, until a clear mixture was formed. Next, Labrasol was introduced, followed by gentle agitation ( $40 \mathrm{r} / \mathrm{min}$ ) for about 1 hour until the mixed micelles formed. Subsequently, $20 \mathrm{~mL}$ of prewarmed distilled water was added, and minutes later, the solution was evaporated in a rotator evaporator at $50 \mathrm{rpm}, 50^{\circ} \mathrm{C}$, and under a vacuum of $-0.1 \mathrm{MPa}$, to remove the alcohol. To obtain the micelles, the sample was subjected to centrifugation at $13,000 \mathrm{rpm}$ for 15 minutes, and the cellulose acetate filters (pore size: $0.45 \mu \mathrm{m}$ ) were filtrated to remove the insoluble substances. A portion of the filtrate was stored for further application or characterization.

\section{Determination of the particle size and} zeta potential of the micelles

The particle size and zeta potential of the prepared micelles were determined by dynamic light scattering using a Zetasizer Nano-DTS 1060 (Malvern Instruments Ltd, Malvern, UK). The measurements were performed at a scattering angle of $90^{\circ}$, after 5 minutes of equilibration of the micelles solution. The temperature was maintained at $25^{\circ} \mathrm{C}$ during the measurements. All of the results were the average of at least three independent samples.

\section{Morphological characterization}

Transmission electron microscopy (TEM) is one of the most common methods used to characterize the morphology of nanoscopic and mixed micelles. The morphology of the prepared micelles in water was observed using TEM (JEM-1200EX; JEOL Ltd, Tokyo, Japan). One drop of the diluted micelles (PPD-phospholipid complex/Labrasol 1:3) 
was placed on a copper grid with nitrocellulose covering. Prior to observation, the sample was negatively stained with $2 \%(\mathrm{w} / \mathrm{v})$ phosphotungstic acid for 5 minutes and dried at room temperature.

\section{Differential scanning calorimetry (DSC)}

The physicochemical properties of the PPD in micelles were evaluated with differential scanning calorimetry. Prior to testing, the solid sample was dried at $40^{\circ} \mathrm{C}$ to remove residual water. The analysis of the PPD, phospholipid, Labrasol, the physical mixture, and mixed micelles was performed using a nitrogen atmosphere DSC (DSC-60; Shimadzu Corp, Kyoto, Japan), and each sample was heated from $10^{\circ} \mathrm{C}$ to $500^{\circ} \mathrm{C}$ at an increasing temperature speed of $10^{\circ} \mathrm{C}$ per minute.

\section{Drug loading (DL) and drug entrapment efficiency (EE)}

DL and EE were measured using a high-performance liquid chromatography (HPLC) method. The HPLC system was an Agilent 1200 system (Agilent Technologies, Santa Clara, CA, USA) equipped with a UV detector set at $203 \mathrm{~nm}$. The separation was performed on a RP-C18 column (Alltech Alltima; Grace, Columbia, MD, USA; $5 \mu \mathrm{m}$, $4.6 \mathrm{~mm} \times 250 \mathrm{~mm}$ ). The mobile phase of the acetonitrile and deionized water $(90 / 10, \mathrm{v} / \mathrm{v})$ was used at a flow rate of $1.0 \mathrm{~mL} /$ min, and the column temperature was maintained at $30^{\circ} \mathrm{C}$. The EE and DL were measured as follows: Briefly, the prepared micelles were dissolved in ethanol and then centrifuged at 13,000 rpm for 15 minutes to obtain the supernatant. The amount of PPD in the resulting solution was then analyzed by HPLC. Each sample was measured three times. The DL and $\mathrm{EE}$ were calculated using the following equations:

$$
\begin{aligned}
\mathrm{EE}(\%)= & \text { Amount of drug in micelles/ } \\
& \text { Amount of drug added; }
\end{aligned}
$$

DL $(\%)=$ Amount of drug in micelles/ Amount of phospholipid complex + Labrasol.

\section{Solubility measurements}

The water solubility was determined by shaking excess solute in water. Excess amounts of the mixed micelles were then dispersed in $2 \mathrm{~mL}$ of water and were shaken at a speed of $40 \mathrm{r} / \mathrm{min}$ for 24 hours, in a heated water bath at $37^{\circ} \mathrm{C}$, until the solution reach a balance. The solution was then centrifuged at 13,000 rpm for 15 minutes and filtered using a $0.45 \mu \mathrm{m}$ membrane. The solubility of free PPD was measured with the same method. The PPD amount in the saturated solution was determined by HPLC, as previously described.

\section{In vitro release studies}

The in vitro release of PPD from the mixed micelles under sink conditions was investigated using dialysis in a simulated intestinal medium, with the addition of $0.5 \%$ Tween $^{\circledR}-80$ (AMRESCO LLC, Solon, OH, USA) as the release medium. Briefly, $5 \mathrm{~mL}$ of prepared micelles solution was added into a dialysis bag (molecular weight cut-off [MWCO] $=6000$ 8000 Da; Spectrum Laboratories Inc, Rancho Dominguez, CA, USA), and the dialysis bag was placed into the release medium, with a paddle revolution speed of $100 \mathrm{rpm}$, at $37^{\circ} \mathrm{C}$. At specific time intervals, $2 \mathrm{~mL}$ of the medium was withdrawn for sample analysis and replaced with an equal volume of prewarmed fresh media. The concentration of the released PPD was determined by HPLC, and the cumulative release profile of the drug at each time point was verified. Each experiment was performed in triplicate.

\section{Cell culture and transport studies through Caco-2 cells}

The human cloned Caco-2 TC7 cells were kindly donated by Dr Ming $\mathrm{Hu}$ of INSERM U178 (Houston, TX, USA). The Caco- 2 cells were seeded on to polycarbonate inserts in Transwell ${ }^{\circledR}$ 6-well plates (growth area, $4.2 \mathrm{~cm}^{2}$ ) at a density of 100,000 cells $/ \mathrm{cm}^{2}$ and grown in Dulbecco's modified Eagle's medium ([DMEM] pH 7.4) supplemented with $10 \%$ fetal bovine serum, which was changed every other day. The Caco-2 cells were incubated in a humidified atmosphere of $5 \% \mathrm{CO}_{2} / 95 \%$ air at $37^{\circ} \mathrm{C}$. Transport studies were performed with the cell monolayers, which were cultured for 21-23 days.

Prior to the transport studies, transepithelial electrical resistance (TEER) was measured as a marker of the integrity of each monolayer. The Caco- 2 cell monolayer was only used in the experiments if the TEER value of the cell monolayer was greater than $300 \Omega / \mathrm{cm}^{2}$. After the culture medium was removed by aspiration, the Caco- 2 cell monolayer was washed three times with $37^{\circ} \mathrm{C} \mathrm{HBSS}(\mathrm{pH} 7.4)$, and the monolayer was incubated for 1 hour in $\operatorname{HBSS}$ at $37^{\circ} \mathrm{C}$ with 2.5 and $2.9 \mathrm{~mL}$ of the incubation medium on the apical and basal sides, respectively. After the medium was removed, the incubation medium-containing PPD and its micelles solution were added to either the apical $(2.5 \mathrm{~mL})$ or basal side $(2.9 \mathrm{~mL})$. A control vehicle incubation medium was added to the opposite side (basal, $2.9 \mathrm{~mL}$; apical, $2.5 \mathrm{~mL}$ ), and the Transwell culture plate was placed in a shaker at $50 \mathrm{rpm}$ and $37^{\circ} \mathrm{C}$. Transport from the apical side to basal side: HBSScontaining compounds were added to the apical side and (control) HBSS was added to the basal side. Transport from 
the basal side to apical side: HBSS-containing compounds were added to the basal side and HBSS was added to the apical side. Donor $(400 \mu \mathrm{L})$ and receiver samples $(400 \mu \mathrm{L})$ were obtained at intervals of 1,2,3, and 4 hours, followed by the addition of $400 \mu \mathrm{L}$ drug donor solution to the donor side or $400 \mu \mathrm{L}$ of blank buffer to the receiver side. The amount of transported PPD was then determined by HPLC. Following the transport studies, the TEER value was remeasured to confirm the integrity of the cell monolayer.

\section{In vivo studies}

Male Sprague Dawley (SD) rats were obtained from the SLEK Lab Animal Center of Shanghai (Shanghai, China), and the rats were housed in a 12-hour light and 12-hour dark cycle. The rats had access to food and water ad libitum. The animal tests were performed in accordance with the guidelines of the Institution of Animal Care and Use Committee of Jiangsu Provincial Academy of Chinese Medicine (Nanjing, China). All of the animal studies were approved by the Animal Ethics Committee of Jiangsu Provincial Academy of Chinese Medicine.

Twelve healthy SD rats weighing 200 to $250 \mathrm{~g}$ were randomly divided into two groups: intragastric administration of free PPD (Group A); and intragastric administration of mixed micelles (Group B). Prior to the experiment, the rats were fasted for 12 hours but were allowed free access to water. Afterwards, the rats were administered free PPD and preparations of micelles (equivalent to $4 \mathrm{mg} / \mathrm{kg}$ of PPD) intragastrically. Next, $1.5 \mathrm{~mL}$ of blood was collected through orbital sinus bleeding, into heparin-containing tubes at $0,0.25$, $0.5,1,1.5,2,3,4,6,8,12$, and 24 hours after oral administration. The heparinized blood samples were then separated by centrifugation at $4000 \mathrm{rpm}$ for 15 minutes, in a refrigerated centrifuge, and the supernatant sample of blood plasma was separated and transferred into $2 \mathrm{~mL}$ centrifuge tubes. The plasma samples were stored at $-20^{\circ} \mathrm{C}$ until further analysis.

Prior to the analysis, a $100 \mu \mathrm{L}$ aliquot of plasma sample (stored previously) was added into the centrifuge tube in addition to $20 \mu \mathrm{L}$ of internal standard solution (ginsenoside-Rh2, $50 \mathrm{ng} / \mathrm{mL}$ ), followed by vortexing for 1 minute. Next, $900 \mu \mathrm{L}$ of ethyl acetate was added into each tube and vortex-mixed for 5 minutes, to fully precipitate the protein and extract PPD from the plasma sample. Next, the samples were centrifuged at $13,000 \mathrm{rpm}$ for 15 minutes to obtain the supernatant, which was then transferred into another tube and evaporated to dryness at $25^{\circ} \mathrm{C}$, using a Thermo Savant SPD 2010 Speed Vac System (Thermo Fisher Scientific, Waltham, MA, USA). The residue was dissolved in $200 \mu \mathrm{L}$ of methanol and centrifuged for 15 minutes at $13,000 \mathrm{rpm}$. The supernatant $(20 \mu \mathrm{L})$ was injected into an HPLC-mass spectrometer for analysis. The pharmacokinetic parameters, including maximum concentration $\left(\mathrm{C}_{\max }\right)$, time to peak concentration $\left(\mathrm{T}_{\max }\right)$, half-life $\left(\mathrm{t}_{1 / 2}\right)$, mass/retention time (MRT), $\mathrm{AUC}_{0-\mathrm{t}}$ and $\mathrm{AUC}_{0-\infty}$ were calculated by compartmental analysis using the software program DAS (v 1.0; Consoft Systems Pvt Ltd, Hyderabad, India).

\section{Chromatographic conditions and mass spectrometric conditions}

Separation was performed on a SB-C18 analytical column (Zorbax extend, $150 \mathrm{~mm} \times 2.1 \mathrm{~mm}$ id, $5 \mu \mathrm{m}$; Agilent) with a mobile phase of methanol and water $(95: 5, \mathrm{v} / \mathrm{v})$, at a flow rate of $0.4 \mathrm{~mL} / \mathrm{min}$. The mass spectrometer was operated in the positive ion mode. In the atmospheric-pressure chemical ionization (APCI) source mode, the probe was set at $4.0 \mathrm{kV}$, and the temperature of the probe was maintained at $350^{\circ} \mathrm{C}$. The nitrogen served as a nebulizing gas (flow rate: $1.0 \mathrm{~L} /$ min). Mass spectra were obtained at a dwell time of 0.2 and 1 second in SIM and scan mode, respectively. The masses were scanned from $\mathrm{m} / \mathrm{z} 300$ to 500, where the major fragment ions were detected. The SIM mode positive ions of PPD and $\mathrm{Rh}_{2}$ were monitored at an $\mathrm{m} / \mathrm{z}$ of 425 and 318 , respectively.

\section{Data analysis}

The transport rate through the Caco-2 cell monolayer was expressed as the apparent permeability coefficient $\left(\mathrm{P}_{\text {app }}\right)$ and was calculated using the following equation, as reported by Artursson and Karlson: ${ }^{23}$

$$
\mathrm{P}_{\mathrm{app}}=\frac{\mathrm{V}}{\mathrm{S} \times \mathrm{C}} \times \frac{\mathrm{dC}}{\mathrm{dt}}=\frac{1}{\mathrm{~S} \times \mathrm{C}} \times \frac{\mathrm{dM}}{\mathrm{dt}},
$$

where $\mathrm{V}$ is the volume of the receiver, $\mathrm{S}$ is the surface area of the cell monolayer, $\mathrm{C}$ is the initial concentration, $\mathrm{dC} / \mathrm{dt}$ is the rate of concentration change on the receiver side, and $\mathrm{dM} / \mathrm{dt}$ is the rate of drug transport.

\section{Statistical analysis}

The statistical analysis of the samples was performed using a Student's $t$-test. All of the data were represented as the mean \pm standard deviation (SD). The experiments were performed in triplicate unless otherwise stated, and $P<0.05$ was considered statistically significant.

\section{Result}

Particle size and zeta potential of micelles The particle size and zeta potential are important indices for micelles. The average particle size and zeta potential of the 
micelles at different weight ratios of the PPD-phospholipid complexes and Labrasol are presented in Table 1. An increase in the relative amount of Labrasol to PPD-phospholipid complex resulted in a clear decrease in the particle size and zeta potential. When the ratio reached $1: 3$, the particle size demonstrated an average distribution of $90.5 \pm 0.8 \mathrm{~nm}$, and the micelles solution was negatively charged, with a mean zeta potential of approximately $-28.6 \pm 0.2 \mathrm{mV}$. The high absolute value of the zeta potential indicated that the micelles solution demonstrated good stability. Small particle sizes and high zeta potentials contribute to the stability of the micelles following oral administration. Thus, in the following transport and pharmacokinetic studies, we used mixed micelles with small particle size and high zeta potential.

\section{Morphological characterization}

Using TEM, we performed a morphological study of the prepared PPD-enriched micelles and showed that the micelles exhibited a regular spherical or spheroid morphology, with an average diameter of approximately $42.2 \pm 0.3 \mathrm{~nm}$. These measurements were significantly different from the results obtained with the spectrometer. This difference was mainly due to water evaporation in the electron microscopy experiments, where the micelles exhibited a dry state and lacked a hydration layer, thus resulting in their smaller size. The TEM image is shown in Figure 2.

\section{DSC}

DSC is a rapid and reliable technique and was used to investigate the physical status of the PPD inside the micelles. DSC thermograms of different samples (PPD, phospholipid, Labrasol, physical mixture, and the micelles) are shown in Figure 3. The DSC curve of the PPD (Figure 3A) exhibited a sharp endothermic peak at $214.5^{\circ} \mathrm{C}$, which corresponds to its melting point, indicating its crystalline nature. This was followed by an exothermic peak at $448.9^{\circ} \mathrm{C}$, which

Table I Mean particle size, zeta potential, and PDI of the micelles with different mass ratios of PPD-phospholipid complex and Labrasol ${ }^{\circledR}$ (Gattefossé, St-Priest, France)

\begin{tabular}{llll}
\hline $\begin{array}{l}\text { Micelles (ratio of } \\
\text { PPD-phospholipid } \\
\text { complex and } \\
\text { Labrasol) }\end{array}$ & $\begin{array}{l}\text { Average size } \\
(\mathbf{n m})\end{array}$ & $\begin{array}{l}\text { Zeta potential } \\
(\mathbf{m V})\end{array}$ & PDI \\
\hline I:I & & & \\
I:3 & $132.6 \pm 8.2$ & $-14.2 \pm 3.9$ & $0.251 \pm 0.023$ \\
I:6 & $90.5 \pm 1.8$ & $-28.6 \pm 1.2$ & $0.186 \pm 0.015$ \\
I:9 & $68.7 \pm 1.2$ & $-30.5 \pm 2.1$ & $0.169 \pm 0.019$ \\
\hline
\end{tabular}

Note: $\mathrm{n}=3$.

Abbreviations: PPD, 20(S)-protopanaxadiol; PDI, protein dispersibility index.

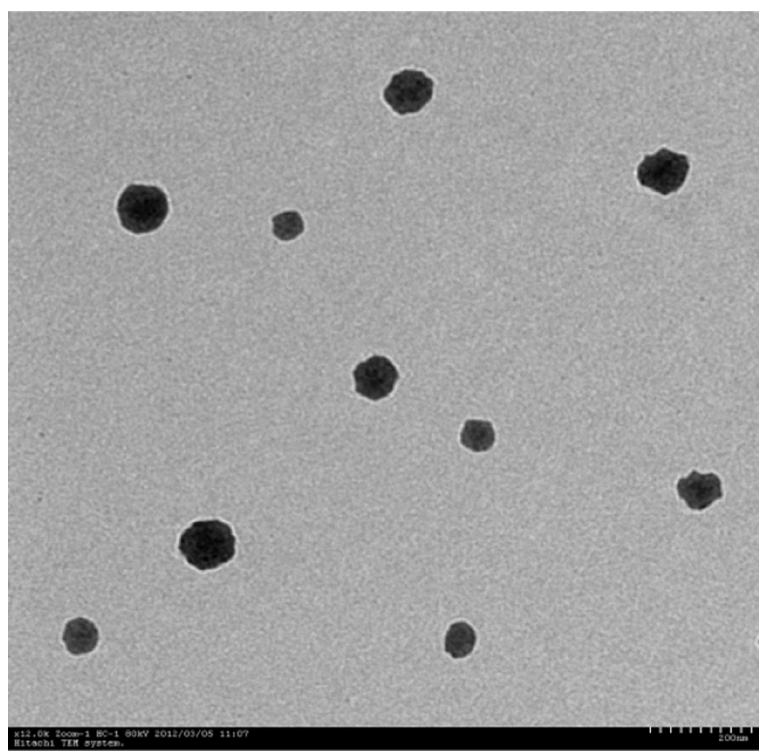

Figure 2 TEM image of prepared micelles in water. Abbreviation: TEM, transmission electron microscopy.

might be attributed to the partial pyrolysis of PPD. However, the phospholipids did not demonstrate sharp endothermal peaks, and the Labrasol exhibited only one sharp endothermic peak at approximately $350^{\circ} \mathrm{C}$. The thermogram of the physical mixture showed all of the endothermic peaks of PPD, phospholipids, and Labrasol, which indicated that the physical mixture did not affect the structure of each sample. As shown in Figure 3E, the DSC thermogram of the micelles showed only one endothermic peak corresponding to Labrasol, and the endothermic peaks corresponding to PPD had disappeared. These results demonstrated that the PPD was encapsulated inside the mixed micelles in a molecular or amorphous state, resulting in the lack of free drug on the micelle surfaces.

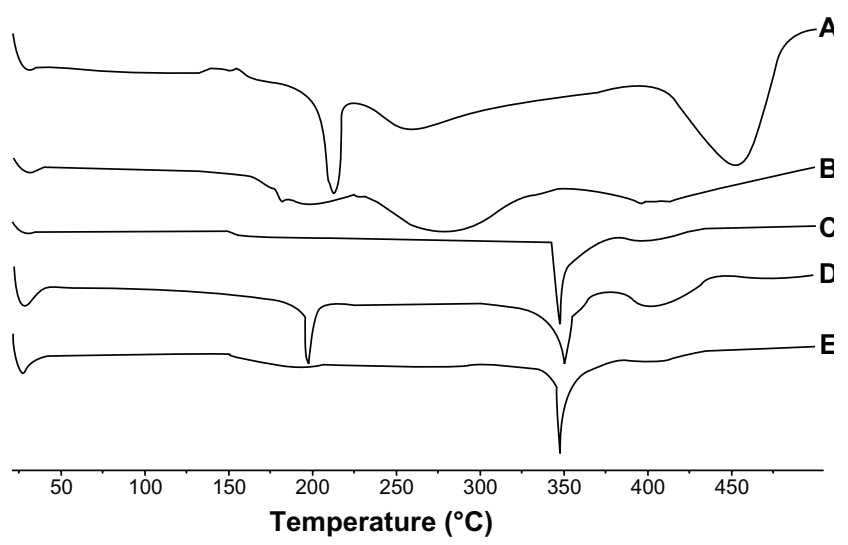

Figure 3 DSC analysis of (A) PPD; (B) phospholipid; (C) Labrasol ${ }^{\circledR}$ (Gattefossé, St-Priest, France); (D) physical mixture; and (E) the micelles (I:3). Abbreviations: DSC, differential scanning calorimetry; PPD, 20(S)-protopanaxadiol. 


\section{$\mathrm{DL}$ and $\mathrm{EE}$}

DL and EE are important indices to evaluate whether or not the PPD is suitable for preparation into the micelles. The DL of the mixed micelles (1:3) was $17.24 \% \pm 2.09 \%$, and EE of the prepared micelles was $93.53 \% \pm 2.28 \%$, as shown in Table 2 . These results suggest that the encapsulation of PPD in the mixed micelles system was high at this DL, but the DL of high proportion was too low. Therefore, considering the particle size and the amount of DL, we selected the mixed micelles (1:3).

\section{Solubility measurements}

To evaluate whether any increase in drug solubility occurred in the presence of the mixed micelles, solubility studies of the PPD-enriched micelles were performed, and the results are shown in Table 2. The solubility of the PPD in the mixed micelles was significantly higher than that of free PPD. In addition, the solubility of the PPD in the mixed micelles was $89.82 \pm 1.13 \mu \mathrm{g} / \mathrm{mL}(1: 1)$ and $192.41 \pm 2.58 \mu \mathrm{g} / \mathrm{mL}(1: 3)$ at different mass ratios of Labrasol. The water solubility of the PPD was clearly increased, by approximately 64-fold, compared with the water solubility of free PPD $(3 \mu \mathrm{g} / \mathrm{mL})$ at room temperature, which demonstrates that the micelles $(1: 3)$ could significantly increase the solubility of PPD. This was mainly due to the structure of Labrasol, which caused stronger hydrophobic interactions between the PPD-phospholipid complex and Labrasol.

\section{In vitro release studies}

The in vitro release was measured to evaluate the promoting effects of the mixed micelles on the in vitro release. The release behaviors of free PPD and the micelles in the simulated intestinal medium under sink conditions are shown in Figure 4, which shows that free PPD was released more rapidly, while the mixed micelles release curve was more

Table 2 Solubility, drug loading, and entrapment efficiency of the mixed micelles with different mass ratios of PPD-phospholipid complex and Labrasol ${ }^{\circledR}$ (Gattefossé, St-Priest, France)

\begin{tabular}{llll}
\hline $\begin{array}{l}\text { Micelles (ratio of } \\
\text { PPD-phospholipid } \\
\text { complex and } \\
\text { Labrasol) }\end{array}$ & $\begin{array}{l}\text { Solubility } \\
(\mu \mathrm{g} / \mathrm{mL})\end{array}$ & EE (\%) & DL (\%) \\
\hline I:I & & & \\
I:3 & $89.82 \pm 1.13$ & $83.28 \% \pm 1.91 \%$ & $17.24 \% \pm 2.09 \%$ \\
I:6 & $192.41 \pm 2.58$ & $93.53 \% \pm 2.28 \%$ & $10.54 \% \pm 1.93 \%$ \\
I:9 & $257.69 \pm 1.94$ & $94.15 \% \pm 1.36 \%$ & $8.71 \% \pm 1.76 \%$ \\
\hline & $336.28 \pm 3.62$ & $92.16 \% \pm 1.89 \%$ & $5.89 \% \pm 1.58 \%$ \\
\hline
\end{tabular}

Note: $\mathrm{n}=3$.

Abbreviations: EE, entrapment efficiency; DL, drug loading; PPD, 20(S)protopanaxadiol. stable. Compared with free PPD, approximately $20 \%$ of the PPD was released from the micelles within the first 12 hours and was continuously released over 1 week. In contrast, more than $90 \%$ of the free PPD was released within 24 hours. These findings suggest that the mixed micelles had a specific, sustained release effect on the in vitro release, possibly due to the high affinity of the PPD and the hydrophobic domain in the micelles, which contributed to its inefficient release from nanoparticles.

\section{Transport through Caco-2 cells}

The Caco-2 cell monolayer was used to investigate drug absorption in this study. The effect of the micelles on the transport via the Caco-2 cell monolayer was measured. The bidirectional transport of free PPD and PPD enriched with micelles through the Caco- 2 cell monolayer was also investigated. These results are shown in Table 3 and Figure 5. The permeability of free PPD and mixed micelles, from the apical to basal side, was $(1.12 \pm 0.21) \times 10^{6} \mathrm{~cm} / \mathrm{s}$ and $(1.78 \pm 0.37) \times 10^{6} \mathrm{~cm} / \mathrm{s}$, respectively, and the permeability of transport in the opposite direction (basal to apical side) of the micelles was $(2.42 \pm 0.16) \times 10^{6} \mathrm{~cm} / \mathrm{s}$ compared with $(2.12 \pm 0.32) \times 10^{6} \mathrm{~cm} / \mathrm{s}$ in the free PPD solution. As a result, at the end of the 4 hours transport study using Caco- 2 cells, the efflux ratio (the ratio of the $\mathrm{P}_{\text {app }}$ basolateral-apical to $\mathrm{P}_{\text {app }}$ apical-basolateral) of the mixed micelles was 1.12 (decrease of $108 \%$ ) compared with 2.16 for the free PPD. Thus, the mixed micelles in this study effectively increased the absorption and inhibited excretion. Before and after the experiment, the average TEER value was $485 \Omega / \mathrm{cm}^{2}$ and $452 \Omega / \mathrm{cm}^{2}$, respectively, which showed that the Caco-2 cell monolayers maintained their integrity during the study.

\section{Pharmacokinetics study in rats}

The method used in this study was successfully applied to quantify the PPD in rat plasma following oral administration $4 \mathrm{mg} / \mathrm{kg}$ PPD and mixed micelles (equivalent to $4 \mathrm{mg} / \mathrm{kg}$ PPD). The mean plasma concentration-time profiles are shown in Figure 6, and the main pharmacokinetic parameters of the PPD are depicted in Table 4. These two curves were both characterized by a rapid increase and subsequent slow decrease.

The average $\mathrm{C}_{\text {max }}$ of PPD was $74.87 \pm 25.38 \mathrm{ng} / \mathrm{mL}$, and this corresponds to the mean $\mathrm{T}_{\max }$ value, which was $90 \pm 7.75$ minutes after oral administration of free PPD. The $\mathrm{C}_{\max }$ of the PPD was $141.87 \pm 63.26 \mathrm{ng} / \mathrm{mL}$ at a $\mathrm{T}_{\max }$ of $90 \pm 22.58$ minutes after oral administration of the mixed micelles. Compared with the PPD, the $t_{1 / 2}$ of the mixed micelles exhibited no significant 


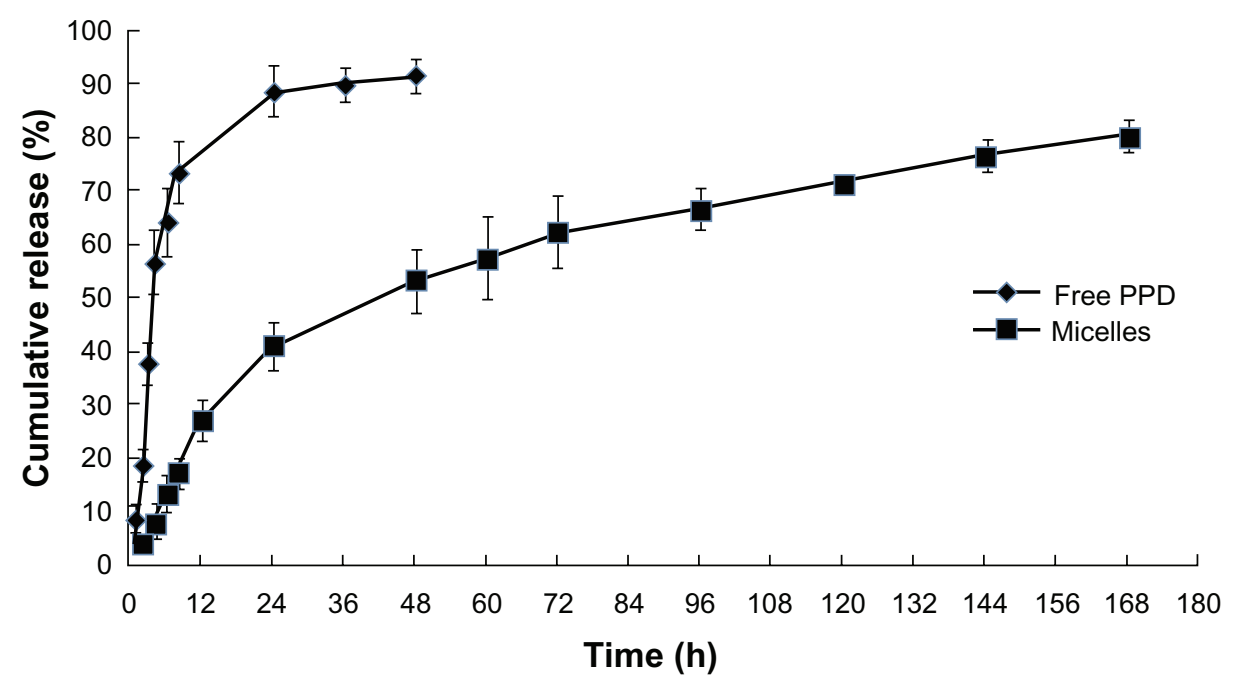

Figure 4 Drug release profiles of free PPD and the mixed micelles (I:3) in the simulated intestinal medium, with addition of $0.5 \%$ Tween ${ }^{\circledR}-80$ (AMRESCO LLC, Solon, OH, USA) as a sink solution at $\mathrm{pH} 6.8$.

Abbreviation: PPD, 20(S)-protopanaxadiol.

change. The average values of the $\mathrm{AUC}_{0-\infty}(\mathrm{mg} / \mathrm{L} \cdot \mathrm{min})$ of the PPD and micelles were $28.41 \pm 822$ and $61.47 \pm 62.39$, respectively, which suggested that novel mixed micelles with PPD-phospholipid complexes and Labrasol could promote absorption and increase relative bioavailability (116.37\% increase). In this study, Labrasol was not only used as a surfactant, but also as an absorption enhancer, which resulted in enhanced absorption and greater bioavailability.

\section{Discussion}

Previous studies have shown that micelles are ideal vehicles for the delivery of hydrophobic drugs. Mixed micelles have a core-shell structure, and poorly soluble drugs may be solubilized within the hydrophobic inner core of the micelles. Thus, micelle delivery can be an effective approach to improve the curative effect of insoluble drugs. Phospholipids are amphoteric compounds and an important component of the cell membrane; they maintain cell membrane fluidity and enable drugs to be easily absorbed. Drug-phospholipid complexes are novel drug-carrier systems that were initially described by the Italian researcher Bombardelli during his studies on

Table 3 Permeability of PPD and the mixed micelles (1:3)

\begin{tabular}{llll}
\hline Sample & $\mathbf{P}_{\text {app }} \times 10^{-6}\left(\mathbf{c m} \cdot \mathbf{s}^{-1}\right)$ & & $\mathbf{P}_{\text {B-A }} / \mathbf{P}_{\text {A-B }}$ \\
\cline { 2 - 3 } & AP-BL & BL-AP & \\
\hline PPD & $1.12 \pm 0.21$ & $2.42 \pm 0.16$ & 2.16 \\
Mixed micelles & $1.78 \pm 0.37^{*}$ & $2.12 \pm 0.32$ & $1.19 *$ \\
\hline
\end{tabular}

Notes: Absorptive permeability was expressed as A-B, whereas secretory permeability was expressed as B-A. Efflux ratio was $P_{\text {app }}(B L-A P) / P_{\text {app }}(A P-B L)$.

The data are presented as mean \pm standard deviation, $\mathrm{n}=3$. $* P<0.05$ versus PPD group.

Abbreviations: AP, apical; BL, basolateral; $\mathrm{P}_{\text {app }}$, apparent permeability coefficient; PPD, 20(S)-protopanaxadiol. liposomes. ${ }^{24}$ The active components of traditional Chinese medicine and phospholipids form phospholipid complexes under specific conditions. As reported, the phospholipid complexes of oxymatrine, baicalin, catechin, puerarin, and quercetin improved gastrointestinal absorption and increased bioavailability. ${ }^{25-29}$ However, the low water solubility of the phospholipid complex limits its application. Fortunately, compared with the free drug, the phospholipid complex easily forms micelles (due to its lipophilic alkyl tails) and can form mixed micelles of phospholipid complexes and the surfactant Labrasol, (which was targeted, in this study, to increase the solubility and permeability of PPD).

In most therapeutic agents, the oral route is still the most convenient for drug administration. The preparation of micelles in studies is similar, and thus it is important to

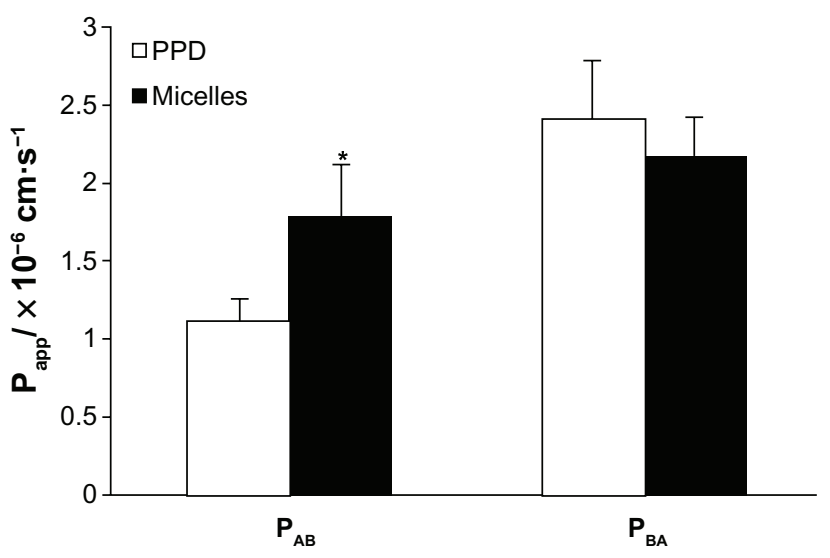

Figure 5 Permeability of PPD and the micelles.

Notes: The data are presented as mean \pm standard deviation, $n=3 . * P<0.05$ versus PPD group.

Abbreviations: $\mathrm{P}_{\text {app }}$, apparent permeability coefficient; $\mathrm{P}_{\mathrm{AB}}$, absorptive permeability; $P_{B A}$, secretory permeability; PPD, 20(S)-protopanaxadiol. 


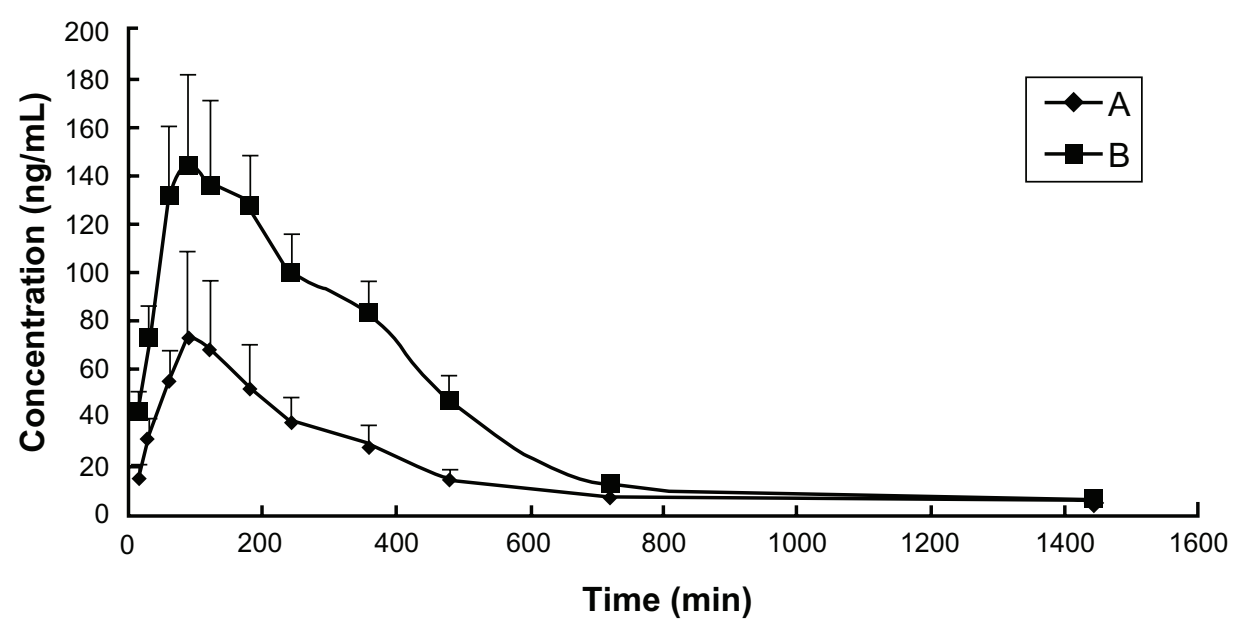

Figure 6 Mean plasma concentration-time profiles of PPD in rats after a single oral administration of (A) PPD and (B) the mixed micelles consisting of PPD- phospholipid complex and Labrasol $^{\circledR}$ (Gattefossé, St-Priest, France).

Notes: The data are presented as mean \pm standard deviation, $n=6$.

Abbreviations: PPD, 20(S)-protopanaxadiol.

choose a good model to investigate its absorption in the gastrointestinal system. There are many different types of test models that can be used to investigate drug absorption in the gastrointestinal system. Although the most reliable results may be obtained using human in vivo experiments, there are different methods available for in vitro and in vivo study. The Caco-2 cell monolayer model, which is routinely used to simulate the drug absorption process, is recognized by the US Food and Drug Administration (FDA) ${ }^{30}$ as a viable model of human small-intestinal absorption, because it exhibits a well-differentiated brush border on its apical surface, tight junctions, and it expresses typical small-intestinal microvillus hydrolases and various cytochrome $\mathrm{P} 450$ isoforms, and P-glycoprotein and phase II enzymes such as UDPglucuronosyltransferases, sulfotransferases, and glutathioneS-transferases. ${ }^{31}$ Several of these transporters promote drug absorption; ${ }^{32}$ however, P-glycoprotein is known to restrict drug

Table 4 Mean pharmacokinetic parameters of PPD after oral administration of PPD $(4 \mathrm{mg} / \mathrm{kg}$ ) and the mixed micelles (equivalent to $4 \mathrm{mg} / \mathrm{kg}$ PPD) in six Sprague Dawley rats

\begin{tabular}{lcr}
\hline Parameters & \multicolumn{1}{c}{ PPD } & Micelles (I:3) \\
\hline $\mathrm{AUC}_{0-\mathrm{t}}(\mathrm{mg} / \mathrm{L} \cdot \mathrm{min})$ & $25.79 \pm 9.34$ & $59.14 \pm 51.29$ \\
$\mathrm{AUC}_{0-\infty}(\mathrm{mg} / \mathrm{L} \cdot \mathrm{min})$ & $28.41 \pm 8.22$ & $61.47 \pm 62.39$ \\
$\mathrm{MRT}_{0-\mathrm{t}}(\mathrm{min})$ & $361.18 \pm 49.84$ & $322.48 \pm 77.39$ \\
$\mathrm{MRT}_{0-\infty}(\mathrm{min})$ & $489.76 \pm 43.74$ & $360.29 \pm 85.38$ \\
$\mathrm{t}_{1 / 2}(\min )$ & $373.73 \pm 30.49$ & $363.87 \pm 30.93$ \\
$\mathrm{~T}_{\max }(\mathrm{min})$ & $90 \pm 7.75$ & $90 \pm 22.58$ \\
$\mathrm{C}_{\max }(\mathrm{ng} / \mathrm{mL})$ & $74.87 \pm 25.38$ & $141.87 \pm 63.26$ \\
\hline
\end{tabular}

Notes: The data are presented as mean \pm standard deviation, $n=6$.

Abbreviations: AUC, area under the curve; $C_{\text {max }}$, maximum concentration; MRT, mass/retention time; PPD, 20(S)-protopanaxadiol; $t_{1 / 2}$, half-life; $T_{\text {max }}$ time to peak concentration. absorption in the intestinal mucosa. ${ }^{33}$ The Caco-2 cell lines have been previously used in oral absorption studies, and it has been established as the most popular in vitro model to evaluate drug transport. ${ }^{34,35}$ In addition, another model was performed in rats, which may better simulate the absorption in vivo.

Labrasol (Caprylocaproyl macrogol-8 glycerides) is an oiland water-soluble liquid. The chemical structure of Labrasol exhibits both a hydrophilic group and lipophilic allyl, resulting in amphiphilic properties. Its characteristic structure and low toxicity also make it a good nonionic surfactant in oral drug formulations. Moreover, Labrasol has been extensively used for the solubilization of hydrophobic drugs, as an emulsifier and absorption enhancer. ${ }^{36}$ More recently, it was reported that Labrasol exhibits a strong absorption-enhancing effect and that intestinal absorption of different poorly absorbable drugs, including gentamycin, insulin, and vancomycin, was improved in its presence. ${ }^{37-39}$ It has been demonstrated that Labrasol might inhibit the function of P-glycoprotein in the intestine, which inhibits excretion and increases intestinal absorption and bioavailability. ${ }^{40}$ In this study, we developed mixed micelles consisting of PPD-phospholipid complexes and Labrasol, to increase the water solubility and permeability of PPD. In the mixed micelles, PPD-phospholipid complexes were formed between the PPD C-OH and phospholipids via hydrogen bond interactions or van der Waals forces and the structure of polyethylene glycol in Labrasol (via its hydrophobic segments). This results in the aggregation at its core, making it hydrophilic. Thus, poorly soluble drugs can be made soluble within the hydrophobic inner core of a micelle and the hydrophilic shell interface of the biological media. As a 
result, micelles can substantially improve the solubility and bioavailability of various hydrophobic drugs.

The drug-release profiles in Figure 4 suggest that no more than $90 \%$ of PPD from the micelles was released in a week, which indicates a very slow release. This might be due to the fact that PPD and phospholipid could form the hydrophobic core of the micelles and that the hydrophobic tails of PPDphospholipid complexes were combined with the lipophilic chains of Labrasol. Therefore, there existed a good affinity between PPD and the hydrophobic group of phospholipid and Labrasol; meanwhile, the polyethylene glycol chains of Labrasol could also block drug release from the micelles. These results are consistent with the work reported by Ganta et al. ${ }^{41}$ The transport studies of the mixed micelles showed that in the same concentration, the permeability of the micelles was greater than that of the free PPD solution, and the ratio of the $\mathrm{P}_{\text {app }}$ basolateral-apical to $\mathrm{P}_{\text {app }}$ apical-basolateral (efflux radio) was largely reduced (from 2.16 to 1.19 ) compared with the free PPD solution. This indicates good absorption, and similar results were found in the pharmacokinetics study performed in rats. We estimated that the enhanced bioavailability of PPD was more likely due to facilitated absorption by the formation of micelles rather than an improved release. This significant absorption-promoting effect may be attributed to micelles that were absorbed by the adsorptive endocytosis pathway. ${ }^{42}$ The internalization of the carrier may have been triggered by nonspecific interactions between the intestinal cells and micelles. ${ }^{43}$ In addition, the micelles may have been absorbed by fluid-phase pinocytosis, which also results in the membrane invagination and vesicle formation.

Although it exhibits low water solubility and absorptive permeability, PPD is still a potential anticancer drug. In the present study, novel mixed micelles with PPD-phospholipid complexes and Labrasol were prepared using the solventevaporation method. These functional micelles provided several clear advantages, such as excellent solubility of hydrophobic drugs, extended circulating properties, good absorption, and bioavailability in vivo. Furthermore, the mean particle size of the micelles was $90.5 \pm 0.8 \mathrm{~nm}$, and the average zeta potential was $-28.6 \pm 0.2 \mathrm{mV}$. The smaller size and higher zeta potential values indicate that the micelles would be more easily absorbed and more stable. In addition, during the preparation of the micelles, the particle size, zeta potential, drug loading, encapsulation efficiency, and solubility can be adjusted by varying the weight ratio of the PPD, phospholipids, and Labrasol. Thus, the micelles are capable of delivering drugs into poorly permeable tumors and cancers. ${ }^{44}$ In addition, novel mixed micelles consisting of phospholipid complexes and Labrasol increase water solubility, enhance absorption, and improve bioavailability.

\section{Conclusion}

In this study, mixed micelles consisting of PPD-phospholipid complexes and Labrasol were successfully prepared using the solvent-evaporation method. The maximum solubility value of the PPD in the micelles was at least 64 times greater than the water solubility of free PPD. The small particle size and high zeta potential indicated that the micelles were easily absorbed and more stable. The high DL and drug EE confirmed its suitability for micelle preparation. Endothermal peaks in the DSC graphs of the mixed micelles suggested that the PPD was encapsulated in the micelles. The micelles exhibited a slow release compared with the free drug. Transport studies and in vivo studies performed in rats demonstrated that the prepared micelles increased permeability and relative bioavailability. Taken together, the micelles system, consisting of PPD-phospholipid complexes and Labrasol, significantly increased solubility, enhanced absorption, and improved bioavailability. As a result, this study demonstrates the potential of micelles as a novel delivery vehicle.

\section{Acknowledgments}

This work was financially supported by the National Natural Science Foundation of China (No 30572372, No 30973944) and the Open Project of Key Laboratory of Oral Drug Delivery System of State Administration of Traditional Chinese Medicine (2011NDDCM01001).

\section{Disclosure}

The authors report no conflicts of interest in this work.

\section{References}

1. Cui JF, Garle M, Lund E, Björkhem I, Eneroth P. Analysis of ginsenosides by chromatography and mass spectrometry: release of 20 S-protopanaxadiol and 20 S-protopanaxatriol for quantitation. Anal Biochem. 1993;210(2):411-417.

2. Attele AS, Wu JA, Yuan CS. Ginseng pharmacology: multiple constituents and multiple actions. Biochem Pharmacol. 1999;58(11):1685-1693.

3. Bae EA, Han MJ, Choo MK, Park SY, Kim DH. Metabolism of 20(S)and 20(R)-ginsenoside $\mathrm{Rg} 3$ by human intestinal bacteria and its relation to in vitro biological activities. Biol Pharm Bull. 2002;25(1):58-63.

4. Bae EA, Han MJ, Kim EJ, Kim DH. Transformation of ginseng saponins to ginsenoside $\mathrm{Rh} 2$ by acids and human intestinal bacteria and biological activities of their transformants. Arch Pharm Res. 2004;27(1):61-67.

5. Mochizuki M, YooYC, Matsuzawa K, et al. Inhibitory effect of tumor metastasis in mice by saponins, ginsenoside-Rb2, 20(R)- and 20(S)-ginsenosideRg3, of red ginseng. Biol Pharm Bull. 1995;18(9):1197-1202.

6. Iishi $\mathrm{H}$, Tatsuta M, Baba M, et al. Inhibition by ginsenoside Rg3 of bombesin-enhanced peritoneal metastasis of intestinal adenocarcinomas induced by azoxymethane in Wistar rats. Clin Exp Metastasis. 1997;15(6):603-611.

7. Ota T, Maeda AM, Odashima S. Mechnism of action of ginsenoside Rh2: uptake and metabolism of ginsenoside $\mathrm{Rh} 2$ by cultured B16 melanoma cells. J Pharm Sci. 1991;80(12):1141-1146. 
8. Li W, Liu Y, Zhang JW, et al. Anti-androgen-independent prostate cancer effects of ginsenoside metabolites in vitro: mechanism and possible structure-activity relationship investigation. Arch Pharm Res. 2009;32(1): 49-57.

9. Lee SJ, Ko WG, Kim JH, Sung JH, Moon CK, Lee BH. Introduction of apoptosis by a novel intestinal metabolite of ginseng saponin via cytochrome c-mediated activation of caspase-3 protease. Biochem Pharmacol. 2000;60(5):677-685.

10. Popovich DG, Kitts DD. Ginsenosides 20(S)-protopanaxadiol and $\mathrm{Rh} 2$ reduce cell proliferation and increase sub-G1 cells in two cultured intestinal cell lines, Int-407 and Caco-2. Can J Physiol Pharmacol. 2004;82(3):183-190.

11. Hasegawa H, Lee KS, Nagaoka T, et al. Pharmacokinetics of ginsenoside deglycosylated by intestinal bacteria and its transformation to biologically active fatly acid esters. Biol Pharm Bull. 2000;23(3):298-304.

12. Lee JY, Shin JW, Chun KS, et al. Antitumor promotional effects of a novel intestinal bacterial metabolite (IH-901) derived from the protopanaxadioltype ginsenosides in mouse skin. Carcinogenesis. 2005;26(2):359-367.

13. Yu Y, Zhou Q, Hang Y, Bu X, Jia W. Antiestrogenic effect of 20Sprotopanaxadiol and its synergy with tamoxifen on breast cancer cells. Cancer. 2007;109(11):2374-2382.

14. Lipinski CA, Lombardo F, Dominy BW, Feeney PJ. Experimental and computational approaches to estimate solubility and permeability in drug discovery and development settings. Adv Drug Deliv Rev. 2001;46(1-3):3-26.

15. Serajuddin AT. Salt formation to improve drug solubility. Adv Drug Deliv Rev. 2007;59(7):603-616.

16. Stella VJ, Nti-Addae KW. Prodrug strategies to overcome poor water solubility. Adv Drug Deliv Rev. 2007;59(7):677-694.

17. Brewster ME, Loftsson TL. Cyclodextrins as pharmaceutical solubilizers. Adv Drug Deliv Rev. 2007;59(7):645-666.

18. Torchilin VP. Micellar nanocarriers: pharmaceutical perspectives. Pharm Res. 2007;24(1):1-16.

19. Shin HC, Alani AW, Rao DA, Rockich NC, Kwon GS. Multi-drug loaded polymeric micelles for simultaneous delivery of poorly soluble anticancer drugs. J Control Release. 2009;140(3):294-300.

20. Saxena V, Hussain MD. Poloxamer 407/TPGS mixed micelles for delivery of gambogic acid to breast and multidrug-resistant cancer. Int J Nanomedicine. 2012;7:713-721.

21. Rupp C, Steckel H, Müller BW. Solubilization of poorly water-soluble drugs by mixed micelles based on hydrogenated phosphatidylcholine. Int J Pharm. 2010;395(1-2):272-280.

22. Yokoyama M. Polymeric micelles as a new drug carrier system and their required considerations for clinical trials. Expert Opin Drug Deliv. 2010;7(2):145-158.

23. Artursson P, Karlsson J. Correlation between oral drug absorption in humans and apparent drug permeability coefficients in human intestinal epithelial (Caco-2) cells. Biochem Biophys Res Commun. 1991;175(3): $880-885$.

24. Bombardelli E. Phytosome: new cosmetic delivery system. Boll Chim Farm. 1991;130(11):431-438.

25. Yue PF, Yuan HL, Li XY, Yang M, Zhu WF. Process optimization, characterization and evaluation in vivo of oxymatrine-phospholipid complex, Int J Pharm. 2010;387(1-2):139-146.

26. Wu J, Chen D, Zhang R. Study on the bioavailability of baicalinphospholipid complex by using HPLC. Biomed Chromatogr. 1999; 13(7):493-495.

International Journal of Nanomedicine

\section{Publish your work in this journal}

The International Journal of Nanomedicine is an international, peerreviewed journal focusing on the application of nanotechnology in diagnostics, therapeutics, and drug delivery systems throughout the biomedical field. This journal is indexed on PubMed Central, MedLine, CAS, SciSearch ${ }^{\circledR}$, Current Contents ${ }^{\circledR} /$ Clinical Medicine,
27. Pietta P, Simonetti P, Gardana C, Brusamolino A, Morazzoni P, Bombardelli E. Relationship between rate and extent of catechin absorption and plasma antioxidant status. Biochem Mol Biol Int. 1998;46(5): 895-903.

28. Li Y, Pan WS, Chen SL, et al. [Studies on preparation of puerarin phytosomes and their solid dispersions.] Chin Pharm J. 2006;41(15): 1162-1167. Chinese.

29. Maiti K, Mukherjee K, Gantait A, Ahamed HN, Saha BP, Mukherjee PK. Enhanced therapeutic benefit of quercetin-phospholipid complex in carbon tetrachloride-induced acute liver injury in rats: a comparative study. Iran J Pharmacol Ther. 2005;4(2):84-90.

30. Yang HT, Wang GJ. Transport and uptake characteristics of a new derivative of berberine (CPU-86017) by human intestinal epithelial cell line: Caco-2. Acta Pharmacol Sin. 2003;24(12):1185-1191.

31. Xie HT, Wang GJ, Chen M, et al. Uptake and metabolism of ginsenoside $\mathrm{Rh} 2$ and its aglycon protopanaxadiol by Caco-2 cells. Biol Pharm Bull. 2005;28(2):383-386.

32. Dantzig AH, Hoskins JA, Tabas LB, et al. Association of intestinal peptide transport with a protein related to the cadherin superfamily. Science. 1994;264(5157):430-433.

33. Kool M, de Haas M, Scheffer GL, et al. Analysis of expression of cMOAT (MRP2), MRP3, MRP4, and MRP5, homologues of the multidrug resistance-associated protein gene (MRP1), in human cancer cell lines. Cancer Res. 1997;57(16):3537-3547.

34. Coyuco JC, Liu Y, Tan BJ, Chiu GN. Functionalized carbon nanomaterials: exploring the interactions with Caco-2 cells for potential oral drug delivery. Int J Nanomedicine. 2011;6:2253-2263.

35. Kenzaoui BH, Vilà MR, Miquel JM, Cengelli F, Juillerat-Jeanneret L. Evaluation of uptake and transport of cationic and anionic ultrasmall iron oxide nanoparticles by human colon cells. Int $J$ Nanomedicine. 2012;7:1275-1286.

36. Strickley RG. Solubilizing excipients in oral and injectable formulations. Pharm Res. 2004;21(2):201-230.

37. Eaimtrakarn S, Rama Prasad YV, Ohno T, et al. Absorption enhancing effect of Labrasol on the intestinal absorption of insulin in rats. $J$ Drug Target. 2002;10(3):255-260.

38. Hu ZP, Tawa R, Konishi T, Shibata N, Takada K. A novel emulsifier, Labrasol, enhances gastrointestinal absorption of gentamicin. Life Sci. 2001;69(24):2899-2910.

39. Prasad YV, Puthli SP, Eaimtrakarn S, et al. Enhanced intestinal absorption of vancomycin with Labrasol and D-alpha-tocopheryl PEG 1000 succinate in rats. Int J Pharm. 2003;250(1):181-190.

40. Lin Y, Shen Q, Katsumi H, et al. Effects of Labrasol and other pharmaceutical excipients on the intestinal transport and absorption of rhodamine123, a P-glycoprotein substrate, in rats. Biol Pharm Bull. 2007;30(7):1301-1307.

41. Ganta S, Devalapally H, Shahiwala A, Amiji M. A review of stimuliresponsive nanocarriers for drug and gene delivery. $J$ Control Release. 2008;126(3):187-204.

42. Guan M, Zhu QL, Liu Y, et al. Uptake and transport of a novel anticancer drug-delivery system: lactosyl-norcantharidin-associated N-trimethyl chitosan nanoparticles across intestinal Caco-2 cell monolayers. Int $J$ Nanomedicine. 2012;7:1921-1930.

43. Norris DA, Puri N, Sinko PJ. The effect of physical barriers and properties on the oral absorption of particulates. Adv Drug Deliv Rev. 1998,34(2-3):135-154.

44. Torchilin VP. Structure and design of polymeric surfactant-based drug delivery systems. J Control Release. 2001;73(2-3):137-172.

\section{Dovepress}

Journal Citation Reports/Science Edition, EMBase, Scopus and the Elsevier Bibliographic databases. The manuscript management system is completely online and includes a very quick and fair peer-review system, which is all easy to use. Visit http://www.dovepress.com/ testimonials.php to read real quotes from published authors. 